Von den umgesiedelten Familien des Gwüest haben drei Familien landwirtschaftliche Liegenschaften im unteren Teil des Göschenertales käuflich oder pachtweise übernommen.

Schließlich wurden 7 männliche Aussiedler von der Kraftwerkgruppe als Hilfsarbeiter, Straßen- und Wasserwärter übernommen, wo sie ein gesichertes Auskommen haben.

\begin{tabular}{|c|c|c|c|c|}
\hline $\begin{array}{l}\text { Liegenschafts- } \\
\text { besitzer }\end{array}$ & Personen & $\begin{array}{c}\text { Fläche } \\
\mathrm{m}^{2}\end{array}$ & Gebäude & $\begin{array}{c}\text { Entschädigungsbetrag } \\
\text { Fr. }\end{array}$ \\
\hline 11 & 60 & 2279620 & $\begin{aligned} \text { 1o } & \text { Wohnhäuser } \\
28 & \text { Ställe } \\
1 & \text { Gasthaus } \\
1 & \text { Hotel } \\
1 & \text { Kapelle } \\
1 & \text { Schulhaus }\end{aligned}$ & $1806900 .-$ \\
\hline
\end{tabular}

L'USINE ELECTRIQUE DE LA GÖSCHENERALP

L'auteur décrit la vallée de Göschenen, une vallée transversale de la vallée de la Reuss, du point de vue géographique, et cela, comme elle était et ce qu'elle en est aujourd'hui. En 195o environ, il fût décidé l'exploiter la vallée pour la production d'énergie électrique. Dans ce but il fut construit un barrage à l'est de la colonie «Göscheneralp». La colonie fut submergée. Maintenant, il y a là un lac d'accumulation, d'une capacité de 75 mio m³ d'eau. L'évacuation et le nouvel établissement de la population n'a pas occasionné des difficultés. Au «Gwüest», situé au dessous du poste de barrage, de nouvelles colonies ont été crées.

\title{
ÜBERSICHT DER SPRACHEN EUROPAS UND KAUKASIENS
}

\author{
Max Wehrli
}

Vorbedingung für eine Sprachenstatistik Europas ist die genaue Abgrenzung Europas im Osten. Als Ostgrenze unseres Erdteils nehmen wir die westlichen Grenzen der russischen Regionen Tjumen und Kurgan und die Nordwestgrenze Kasachstans an. Im Südosten bildet der Kaukasuskamm die Grenzscheide zwischen Europa und Asien. Da jedoch Nord- und Transkaukasien ethnographisch zusammengehören, ist die Zuteilung des transkaukasischen Gebietes zum europäischen Raum notwendig. In Europa (mit Transkaukasien) werden, von einigen im Aussterben begriffenen Idiomen abgesehen, 95 Sprachen gesprochen, nämlich 49 indogermanische, 11 uralische, 12 altaische, 19 kaukasische, 3 semitische und das Baskische als isoliert dastehende Sprache. Die indogermanische Sprachfamilie umfaßt in Europa 6 Sprachengruppen (die germanische, keltische, romanische, baltische, slawische und iranische Gruppe) und außerdem noch 4 Einzelsprachen( das Neugriechische, Albanische, Armenische und die Zigeunersprache). Die uralische Sprachfamilie besteht aus der finnougrischen Gruppe und dem Nenzischen (Samojedischen). Zur altaischen Familie gehört die hauptsächlich in Asien verbreitete türkische Sprachengruppe wie auch das in Europa durch den kalmückischen Dialekt vertretene Mongolische. Die kaukasische Familie zerfällt in die nordkaukasische und die kleine, aber nach Bevölkerungszahl wichtigere südkaukasische Gruppe. Die hamitosemitische Sprachfamilie ist in Europa nur durch die semitische Gruppe vertreten. Das Baskische endlich ist mit keiner andern Sprache verwandt und scheint der letzte Rest einer untergegangenen Sprachfamilie zu sein.

Die Bevölkerungszahlen der folgenden statistischen Übersicht beruhen auf Zählungen oder Berechnungen, die von 1950 bis 1960 reichen. In der sowjetischen amtlichen 
Statistik beziehen sich die Zahlenangaben in erster Linie auf das Volkstum nach Sitten und Bräuchen oder Traditionen. Neuerdings sind auch die Sprecherzahlen der betreffenden Sprachen in Prozentangaben beigefügt, z. B.: Mordwinen 1285 000, wovon $78,1 \%$ (= 1003 500) mordwinisch sprechen. Die Karatschaier, Balkaren, Kalmücken, Tschetschenen und Inguschen wurden im Jahre 1945 deportiert, aber nach dem Tode Stalins in ihre früheren Heimstätten zurückgebracht. Leider gibt es in einigen Ländern, so in Frankreich, Italien und Spanien, keine amtliche Sprachenstatistik, oder es werden gewisse Sprachen nicht voneinander unterschieden, so z. B. das Hochdeutsche und Niederdeutsche in Deutschland. In Fällen, wo weitverbreitete Sprachen in beständigem Rückgang begriffen sind, wie dies beim Niederdeutschen und Provenzalischen zutrifft, ist auch eine nur annähernd zuverlässige Schätzung der Sprecherzahl unmöglich.

\section{STATISTIK}

Bei Sprachen, die aus Gründen der engeren Verwandtschaft (seltener aus bevölkerungsstatistischen Gründen) auf die gleiche Zeile gesetzt sind, ist folgendes zu beachten: Ist der Unterschied nur mundartlicher Natur, d.h. unbedeutend, dann sind sie durch das Pluszeichen $(+)$ miteinander verbunden, sonst aber je nach dem Grade der Verschiedenheit durch das Komma oder den Strichpunkt von einander getrennt. Die Namen der Sprachgruppen sind in Großbuchstaben gesetzt, die Bevölkerungszahlen in tausend angegeben (1000 $=1$ Million $)$.

Gesamtbevölkerung Europas (mit Transkaukasien): 586 Millionen

Deutsch; niederdeutsch

Jiddisch

Niederländisch + flämisch

36000

Friesisch

Englisch

Schwedisch (8200), dänisch (4500), norwegisch (3500)

Isländisch (17o), färöisch (3o)

\begin{tabular}{rr}
86000 \\
3200 \\
16000 \\
400 \\
53000 \\
16200 \\
gisch (3500) \\
200 \\
\hline GERMANISCH 175000
\end{tabular}

Irisch (60o), schottisch-gälisch (130)

730

Walisisch oder kymrisch (700); bretonisch (800) 1500

\begin{tabular}{|c|c|}
\hline & KELTISCH \\
\hline Französisch, wallonisch; provenzalisch & \\
\hline Katalanisch & \\
\hline Spanisch $(22000)$, portugiesisch + galicisch & $(11000)$ \\
\hline Spaniolisch oder jüdisch-spanisch & \\
\hline Italienisch ( 50000$)$; sardisch (100o) & \\
\hline Rätoromanisch (70), friaulisch (4oo) & \\
\hline Rumänisch + moldauisch & \\
\hline & ROMANISCH \\
\hline
\end{tabular}

\begin{tabular}{|c|c|c|}
\hline Neugriechisch & & 8700 \\
\hline \multirow[t]{2}{*}{ Litauisch (23oo); lettisch (14oo) } & & 3700 \\
\hline & BALTISCH & 3700 \\
\hline \multicolumn{2}{|l|}{$\begin{array}{l}\text { Russisch (89 0oo), weißrussisch (7000), ukra } \\
\text { Polnisch (29 90o), kaschubisch (100) } \\
\text { Sorbisch oder wendisch } \\
\text { Tschechisch (9000), slowakisch (4ooo) } \\
\text { Serbokroatisch (11 000), slowenisch (1500) } \\
\text { Bulgarisch (7600), mazedonisch (1200) }\end{array}$} & $\begin{array}{r}132000 \\
30000 \\
30 \\
13000 \\
12500 \\
8800 \\
\end{array}$ \\
\hline & SLAWISCH & 196330 \\
\hline
\end{tabular}




\begin{tabular}{|c|c|c|}
\hline isch & & $27 c$ \\
\hline \multirow[t]{2}{*}{$\begin{array}{l}\text { Ossetisch } \\
\text { Tatisch (persischer Dialekt) } \\
\text { Talyschisch (kaspisch-iranischer Dialekt) } \\
\text { Kurdisch }\end{array}$} & \multirow[b]{2}{*}{ IRANISCH } & $\begin{array}{r}+0 c \\
+c \\
12 c\end{array}$ \\
\hline & & \\
\hline \multicolumn{2}{|c|}{ Zigeunersprache (zur indischen Gruppe gehörend) } & \\
\hline \multirow{2}{*}{\multicolumn{2}{|c|}{$\begin{array}{l}\text { Finnisch (4ooo), karelisch (200), wepsisch (20); estnisch (980) } \\
\text { Lappisch } \\
\text { Mordwinisch } \\
\text { Mari oder tscheremissisch } \\
\text { Udmurtisch oder wotjakisch (560), Komi oder syrjänisch (+oo) } \\
\text { Ungarisch } \\
\text { FINNOUGRISCH }\end{array}$}} & $\begin{array}{r}520 \\
3 \\
100 \\
+8 \\
96 \\
1170\end{array}$ \\
\hline & & 193 \\
\hline \multicolumn{3}{|l|}{ Nenzisch (samojedisch) } \\
\hline \multicolumn{2}{|c|}{$\begin{array}{l}\text { Tschuwaschisch } \\
\text { Tatarisch }(400) \text {, baschkirisch }(700) \\
\text { Kasachisch } \\
\text { Nogaisch } \\
\text { Kumükisch (160), karatschaiisch }(80)+\text { balkarisch (4o) } \\
\text { Türkisch (3000), aserbeidschanisch }(2850) \text {, turkmenisch (50) } \\
\text { Gagausisch }\end{array}$} & $\begin{array}{ll}1 & 25 \\
5 & 20 \\
& 16\end{array}$ \\
\hline & TÜRKISCH & 129 \\
\hline \multicolumn{2}{|l|}{ Kalmückisch (mongolischer Dialekt) } & \\
\hline \multicolumn{2}{|c|}{$\begin{array}{l}\text { Abchasisch (80), abasinisch (20) } \\
\text { Adyge oder tscherkessisch (80), kabardinisch (17o) } \\
\text { Tschetschenisch (300), inguschisch (70) } \\
\text { Awarisch (300), Andi (30), Dido (5) } \\
\text { Lakisch oder kasikumuchisch } \\
\text { Dargwa oder darginisch } \\
\text { Kürinisch (16o), tabassaranisch (15) + agulisch (15), rutulisch } \\
\quad(10) \text {, tsachurisch (10), dschekisch (10) }\end{array}$} & $\begin{array}{l}10 \\
25 \\
37 \\
33\end{array}$ \\
\hline \multicolumn{2}{|c|}{$\begin{array}{l}\text { Georgisch oder grusinisch (2300), mingrelisch (30o) } \\
\text { Swanisch }\end{array}$} & \\
\hline$S \dot{\mathrm{I}}$ & $\mathrm{KASISCH}$ & 26 \\
\hline \multirow[t]{2}{*}{$\begin{array}{l}\text { Hebräisch } \\
\text { Aissorisch oder neusyrisch (neuaramäisch) } \\
\text { Maltesisch (arabischer Dialekt) }\end{array}$} & & \\
\hline & SEMITISCH & \\
\hline
\end{tabular}

\section{ERKLÄRUNGEN ZU DEN WENIGER BEKANNTEN SPRACHEN}

Abasinisch: Die Abasiner, ein Zweig der Abchasen, leben am Kuban, südlich von Stawropol.

Abchasisch: In der Gegend von Suchumi am Schwarzen Meer (Abchasenrepublik).

Adyge oder tscherkessisch: Im Kubangebiet.

Aissorisch: Die Sprache der «assyrischen»Christen, die größtenteils im mittleren Kurdistan (im türkisch-iranisch-irakischen Grenzgebiet) leben, von wo aus viele nach der Sowjetunion, hauptsächlich nach Transkaukasien, auswanderten. Das Aissorische stammt vom sonst ausgestorbenen, aber im Altertum in Vorderasien weit verbreitet gewesenen Aramäischen ab.

Andi und Dido: In Südwest-Dagestan. 
Aserbeidschanisch: In Transkaukasien (besonders in der Sowjetrepublik Aserbeidschan); auch in Persisch-Aserbeidschan verbreitet.

Awarisch: Im Bergland von West-Dagestan und in der Gegend von Sakataly in Aserbeidschan.

Balkarisch: In der Kabardino-balkarischen Republik im Westkaukasus.

Baschkirisch: Im südlichen Ural (Baschkirenrepublik).

Dargwa (darginisch) : In den Bergen südlich von Machatsch-Kala in Dagestan.

Dschekisch: An der Ostgrenze Dagestans auf aserbeidschanischem Boden.

Friaulisch: In Friaul (Friuli), von der österreichischen Grenze bis an den Golf von Triest.

Friesisch: In der niederländischen Provinz Friesland, in Nordfriesland (von Husum bis an die dänische Grenze) und auf den Nordfriesischen Inseln.

Gagausisch: Zwischen Galatz und der Dnjestr-Mündung. Die Gagausen sind Christen.

Hebräisch: Viele Ostjuden (in Polen und der Sowjetunion) haben sich vom Jiddischen abgewandt und sprechen heute hebräisch.

Inguschisch: Im westlichen Teil der Tschetscheno-inguschischen Republik (am mittleren Kaukasus).

Jiddisch: Die Sprache der Ostjuden (in Polen, Rumänien und der Sowjetunion). Das Jiddische ist ein deutscher Dialekt mit Beimischung hebräischer Wörter und wird mit hebräischen Buchstaben geschrieben.

Kabardinisch: Die Kabardiner bilden den östlichen Zweig der Tscherkessen. Sie wohnen an der Nordseite des westlichen und mittleren Kaukasus (in der Kabardino-balkarischen Republik).

Kalmückisch: Im Steppengebiet westlich von Astrachan.

Karatschaiisch: Am oberen Kuban im Westkaukasus.

Kasachisch: Im an Kasachstan angrenzenden russischen Gebiet zerstreut (besonders in Astrachan und Orenburg). Die Hauptmasse der Kasachen lebt in Kasachstan, also außerhalb Europas (etwa 3,5 Millionen).

Kaschubisch, auch Pomoranisch genannt: Westlich von Danzig, zwischen der Ostsee und Bromberg (Bydgoszcz).

Katalanisch: Das katalanische Sprachgebiet umfaßt die Regionen Katalonien und Valencia, die Balearen, Andorra und die südfranzösische Landschaft Roussillon.

Komi oder syrjänisch: In Nordrußland, zwischen Perm und dem Polarkreis.

Kumükisch: An der Küste von Dagestan.

Kurdisch: Im Bergland Karabach in Aserbeidschan. Das Hauptverbreitungsgebiet des Kurdischen ist Ostanatolien mit benachbarten irakischen und iranischen Gebieten.

Kürinisch: In Südost-Dagestan und einigen aserbeidschanischen Grenzorten.

Lakisch (kasikumuchisch) : Im Zentrum des Berglandes von Dagestan.

Lappisch: Im Norden von Schweden-Norwegen-Finnland und auf der Halbinsel Kola.

Maltesisch: Auf den Inseln Malta und Gozo. Das Maltesische ist ein arabischer Dialekt mit Beimischung italienischer Wörter und wird mit lateinischen Buchstaben geschrieben.

Mari oder tscheremissisch: An der mittleren Wolga (Mari-Republik) und im nördlichen Teil der Baschkiren republik.

Mazedonisch: In Jugoslawisch-, Bulgarisch- und Griechisch-Mazedonien. In Jugoslawien ist das Mazedonische regionale Schriftsprache.

Mingrelisch: Westlich von Kutaissi in Georgien.

Moldauisch: Rumänischer Dialekt in der Landschaft Moldau und in der Moldauischen Sowjetrepublik; wird in der Sowjetmoldau mit kyrillischen Buchstaben geschrieben.

Mordwinisch : Über das mittlere Wolgagebiet zerstreut.

Nenzisch (samojedisch) : Im nordrussischen Tundragebiet.

Nogaisch: In der Steppe nördlich von Grosnyj, am Kuban südlich von Stawropol und in der Dobrudscha bei Konstanza. Die im Flachland der Krim ansäßig gewesenen Nogaier wurden mitsamt den Krimtataren im Jahre 1945 deportiert.

Ossetisch: Im mittleren Kaukasus. Die Osseten sind die Nachkommen der alten Alanen und der letzte Rest der nordiranischen Völker (Skythen), die im Altertum Südosteuropa und das heutige Kasachstan bewohnten.

Provenzalisch: In Südfrankreich vom Golf von Biscaya bis an die italienische Grenze; Sprache mit alter literarischer Tradition und zahlreichen Mundarten. Man unterscheidet mehrere Dialektgruppen oder Hauptmundarten (limousin, auvergnat, dauphinois, alpin, provençal, 
languedocien, rouergat, gascon, bearnais). Dazu kommt im Osten die franko-provenzalische Dialektgruppe, welche den Übergang vom Provenzalischen zum Französischen bildet und in der Westschweiz, im Aostatal, in Savoyen und der südlichen Franche Comté verbreitet ist.

Rätoromanisch: In Graubünden (surselvisch und ladinisch) und in einigen Talschaften der Dolomiten (dolomitenladinisch).

Sardisch: Vom Italienischen stark abweichende Sprache in Sardinien mit zwei Hauptmundarten (logudoresisch in Mittel- und Nordsardinien bis in die Nähe von Sassari, campidanesisch in Südsardinien). Das Sassaresische und das Galluresische im äußersten Norden der Insel gehören zum Italienischen.

Sorbisch oder wendisch: In der Lausitz (besonders in der Gegend von Bautzen).

Spaniolisch: Die Sprache der spanischen Juden (Spaniolen) auf der Balkanhalbinsel, besonders in Thessaloniki und Istanbul.

Swanisch: Im Bergland Swanetien südlich vom Elbrus.

Tabassaranisch, agulisch, rutulisch und tsachurisch: In Südost-Dagestan; dem Kürinischen im Norden und Westen vorgelagerte Randdialekte (in der angegebenen Reihenfolge von Ost nach West).

Talyschisch: In der Gegend von Lenkoran in Aserbeidschan; gehört zu der vom Persischen ziemlich stark abweichenden kaspisch-iranischen Dialektgruppe (in den Landschaften Masanderan, Gilan und Talysch).

Tatarisch: Im Wolgagebiet und im südlichen Ural verbreitet. Die Tataren sind im Mittelalter aus Asien eingewandert und waren zeitweise das herrschende Volk in RuBland.

Tatisch: Persischer Dialekt westlich von Baku, am Ostkaukasus.

Tschetschenisch: In der Gegend von Grosnyj in Nordkaukasien (Tschetscheno-inguschische Republik).

Tschuwaschisch: Im mittleren Wolgagebiet, hauptsächlich in der Tschuwaschenrepublik. Die Tschuwaschen kamen mehrere hundert Jahre früher nach Europa als die übrigen Türkvölker. Ihre Sprache hat sich daher eigenartig entwickelt und weicht vom benachbarten Tatarischen sehr stark ab.

Turkmenisch: Die Turkmenen leben größtenteils in Zentralasien. Im europäischen Teil der Sowjetunion zerstreut, z. B. in Nordkaukasien zwischen dem Terek und dem östlichen Manytsch.

Udmurtisch oder wotjakisch: Zwischen der Wjatka und der Kama (Udmurtenrepublik) ; mit dem Komi (Syrjänischen) nahe verwandt, aber von den westfinnischen Sprachen, auch vom Mordwinischen, sehr stark abweichend.

Walisisch oder kymrisch: In Wales; mit dem Bretonischen verwandt, jedoch vom Irischen und Schottisch-Gälischen sehr verschieden.

Wallonisch: Vom Schriftfranzösischen ziemlich stark abweichende Patoisgruppe in Belgien, die allmählich von der französischen Umgangssprache verdrängt wird.

Wepsisch: Am Südwestufer des Onegasees und südlich davon.

Zigeunersprache: Über fast ganz Europa zerstreut. Die Zigeuner leben teils nomadisch, teils in festen Wohnsitzen (Siedlungen hauptsächlich in Bulgarien, Rumänien und der Slowakei). Sie stammen wahrscheinlich aus dem Hindukuschgebiet und kamen während des Mittelalters über Iran, Kleinasien und Griechenland nach Europa. Ihre Sprache gehört zur indischen Gruppe und enthält auch persische, armenische und griechische Wörter. Sie wird in den meisten Ländern, soweit dies nicht bereits geschehen ist, nach und nach durch die betreffenden Landessprachen abgelöst.

\section{QUELLEN}

M. Cohcn: Les langues du Monde. Paris 1952. A. Dauzat: La géographie linguistique. Paris 1948. A. Dauzat: L'Europe linguistique. Paris 194o. E. Lewy: Der Bau der europäischen Sprachen. Dublin 1942. E. Peruzzi: Saggi di linguistica europea. Salamanca 1958. H. Krahe: Sprachliche Aufgliederung und Sprachbewegungen in Alteuropa. Wiesbaden 1959; - Données statistiques. Strassbourg $1959 \mathrm{ff}$.

\section{DES LANGUES DE L'EUROPE ET DE LA CAUCASIE}

La composition d'une statistique d'après les langues se rend difficile par le fait, que les systèmes de relevés ne sont pas coordonnés soit d'après les langues soit d'après les races. De plus il faut observer les périodes différentes de ces recensements. Les données récentes concernent la période de 1950 à 1960. Il était nécessaire de restreindre cet exposé aux groupes caractéristiques. Les groupes slaves et germaniques occupent le premier rang en absolu et en pourcentage. 